\title{
An event selector for sequential control'
}

HAROLD T, SALIVE, UNIVERSITY OF MICHIGAN DAVID E. CARTER, COLUMBIA UNIVERSITY

A circuit using a stepping switch coupled to a number of rotary selector switches is suggested as a general solution to the problem of programming sequential events automatically. A number of applications are discussed including the programming of visual and auditory stimuli and the recording of responses under several different conditions.

In many experimental situations sequences of events must be programmed to change in a predetermined order on the basis of time or at the occurrence of another event, such as the S's response, a reinforcement, etc. In such instances, the use of a stepping switch coupled to a number of rotary selector switches offers a convenient method of control.

Figure 1 illustrates how the circuit of such an event selector is wired. Using the wiper of the stepping switch as an input, each point on the bank of the stepper is wired to the common of a corresponding rotary selector switch. The outputs $(1,2, \ldots$, and $N)$ of the rotary selector switches are connected in parallel to circuitry which controls the occurrence of the events to be programmed. The position of the stepping switch determines which of the rotary selector switches is in control of the programmed event at any given moment. For example, the experiment may require the programming of a sequence of visual stimuli projected onto a pigeon key. In this case, the outputs $(1,2, \ldots$, and $N)$ are connected to the appropriate terminals on the control panel of an in-line display unit (e.g., GrasonStadler, model E4580). The stimulus order is programmed by setting the rotary selector switches to the appropriate positions before the experimentbegins. The sequence is then presented by applying command pulses to the coil of the stepping switch. When the number of

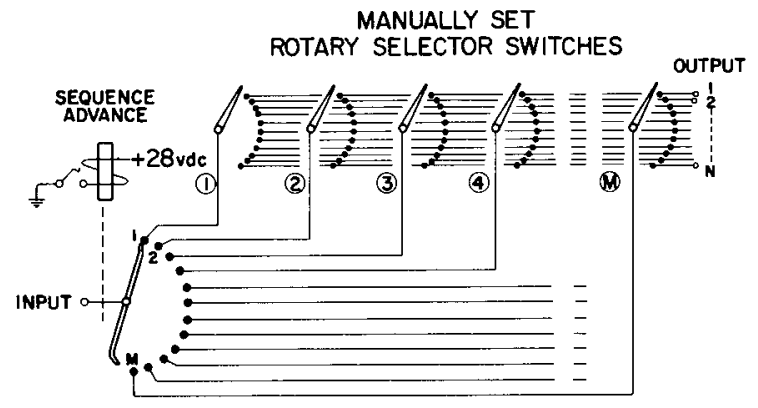

STEPPING SWITCH

Fig. 1. A schematic wiring diagram for a general-purpose event selector. The wiper (common) of an M-point stepping switch serves as the input. Each point on the bank is connected to the common of an N-position rotary selector switch. The like positions of the selector switches are connected in parallel to circuitry which controls the occurrence of the events to be programmed. stimulus presentations exceeds the number of positions available on the stepping switch, the sequence repeats until the experiment is terminated.

of course, the events programmed are not restricted to stimuli. The same apparatus can be used to program any event which either occurs or fails to occur, e.g., as in programming complex reinforcement contingencies. For example, the rotary selector switches can be used to determine which one of several reinforcement schedules is in effect or to program which one of several possible responses will be reinforced on a given trial.

The circuit can also be used for applications in which an input must assume one of a series of possible voltages in some predetermined order. For example, an experiment may require that an auditory stimulus vary in sound pressure level during a generalization test. The auditory signal is passed through a set of fixed attenuators wired in series. The output of each attenuator is wired to a corresponding terminal of each selector switch. When the wiper of the stepping switch is connected to a grounded speaker or pair of earphones, the event selector gives the appropriate sound pressure level at any given moment.

These applications can be combined by duplicating the circuit on an electrically independent but mechanically yoked second level on the stepper and rotary selector switches. One level can then control the stimuli in a generalization test while the second channels responses to the counter associated with the stimulus condition prevailing at the time the response occurs. Additional banks might be used to control a second stimulus dimension as in multidimensional generalization experiments and to terminate the experiment when the predetermined stimulus order has been presented.

We have found it convenient to wire event-selector circuits as permanent units which can be mounted on standard relay racks with the rest of the control circuitry. The high versatility of such units makes them extremely valuable in any laboratory which uses automated switching circuits, especially for experiments which involve stimulus control.

\section{Note}

1. The apparatus described in this paper was developed under grant AF-49(638)-367 from the Air Force Office of Scientific Research to Robert $B$. Zajonc during the period while the second author was a graduate student at the University of Michigan. The authors wish to express their appreciation to John Mills Martin for his critical evaluation of the manuscript. Reprints may be obtained from David E. Carter, 470 Schermerhorn Hall, Department of Psychology, Columbia University, New York, N. Y. 10027. 


\section{Comments on Martin, Cox and Boersma}

by E. A. Lovelace and R. W. Schulz

Recently Martin, Cox, \& Boersma (1965) reported the results of a study in which they attempted to assess the effects of stimulus and response meaningfulness (M) on the associative phase of paired-associate (PA) learning. A recognition method was used in place of the anticipation method because it should "...eliminate response learning and guarantee availability of response items regardless of $M$ values (p. 463)." More specifically, their procedure involved the learning, by the study-test method, of one of the four lists of eight dissyllable pairs representing the four possible combinations of high (H) and low (L) stimulus and response $M$ (H-H, L-H, H-L and L-L). On study trials, pairs were presented at a 3-sec. rate. A multiple-choice arrangement was employed on test trials with each stimulus term along with all eight response items being shown for $4 \mathrm{sec}$. The Ss recorded their response in booklets thereby permitting the use of a group testing procedure. It was found that stimulus $M$, response $M$ and the interaction of these factors were significant sources of variance. Moreover, the effect of response $M$ was greater than that of stimulus $M$.

The present comments are concerned with the effects of response $M$ and the manner in which Martin et al interpret these effects. That is, Martin et al seem to believe that Underwood \& Schulz's (1960) analysis of $M$ in verbal learning implies that elimination of availability differences would lead one to expect, "...the effect due to variation in $M$ on the response side... [ to ] ... be minimized (1965, p. 463)." Quite to the contrary, Underwood and Schulz said, "If the responserecall phase could be completely eliminated, the prediction is that the effect of $M$ on the stimulus and on the response would be equivalent, for both effects would be limited to the associative phase (1960, pp. 95-96)。" Accordingly, the presence of an effect due to response $M$ which is greater than the one due to stimulus $M$ in the Martin et al situation is relevant for the UnderwoodSchulz position if, and only if, response-term availability was, indeed, a minimal factor in their situation. As will be shown below, it seems highly unlikely that this requisite state of affairs prevailed in the Martin et al situation.
At a "purely" empirical level, does the finding of a response-M effect under the present conditions permit the conclusion, "...it appears that [ the effect of ] $M$ on the response side may be attributed to factors other than availability (p. 464)?" To reach this conclusion, one must assume that, independent of response $M$, Ss had time, in 4 sec., to read a dissyllable stimulus, read each of the eight dissyllable responses which accompanied it, and write one of these responses in his booklet. The results of a recent study by the present authors (Schulz \& Lovelace, 1964) strongly suggest that the foregoing assumption may be untenable. In this study the aperture of a memory drum was divided into a 1 in. stimulus-aperture (SA) on the left and an 8 in. response-aperture (RA) on the right. Each member of a set of $10 \mathrm{CVCs}$ of either high or low M appeared alone in SA and at the same timeappeared along with the nine remaining CVCs of that set in RA. Ten pushbuttons were equally spaced below RA, one below each of the $10 \mathrm{CVCs}$. The Ss were simply instructed to find, as rapidly as possible, the item in SA amongst those being presented in RA, and to press the button beneath it. The "arrays" of trigrams were exposed for $5.2 \mathrm{sec}$. The latency with which $\mathrm{S}$ depressed the appropriate button was recorded. The location-latencies were significantly greater for the set of low $M$ trigrams than for high $M$ trigrams.

In conclusion, until it can be shown, perhaps by the method described above, that location-latencies on test trials were equivalent for high and low $\mathrm{M}$ responses in the Martin et al situation, one must interpret their results with appropriate caution regarding the extent to which they reflect, unconfounded by availability differences, the effect of $M$ on the associative phase of PA learning.

\section{References}

Martin, C. J., Cox, D. L., \& Boersma, F. J. The role of associative strategies in the acquisition of P-A material: An alternate approach to meaningfulness. Psychon. Sci., 1965, 3, 463-464.

Schulz, R. W., \& Lovelace, E. A. Meaningfulness and the associative phase of paired-associate learning: A methodological consideration. Psychon. Sci., 1964, 1, 37-38.

Underwood, B. J., \& Schulz, R. W. Meaningfulness and verbal learning. Chicago, Lippincott, 1960. 